\title{
Design and Reflex Control for a Series Elastic Actuator based Ankle Joint Emulator
}

\author{
$1^{\text {st }}$ Mingwen Li \\ School of Automation \\ Wuhan University of Technology \\ Wuhan, China \\ yangfeiyu@whut.edu.cn
}

\author{
$2^{\text {nd }}$ Muye Pang \\ School of Automation \\ Wuhan University of Technology \\ Wuhan, China \\ pangmuye@whut.edu.cn \\ $5^{\text {th }}$ Shenpei Zhou \\ School of Automation \\ Wuhan University of Technology \\ Wuhan, China \\ zhousp@whut.edu.cn
}

\author{
$3^{\text {rd }}$ Kui Xiang \\ School of Automation \\ Wuhan University of Technology \\ Wuhan, China \\ xkarcher@126.com
}

\author{
$4^{\text {th }}$ Zhaojie Ju \\ School of Computing \\ University of Portsmouth \\ Portsmouth, UK \\ zhaojie.ju@port.ac.uk
}

\begin{abstract}
Human body is a highly efficient stable system, although the center of mass is high. While quiet standing ankle joint has the function of resisting disturbance to maintain balance of human body. Studying the control mechanism of ankle joint helps to improve relative technologies such as bipedal robot, powered prosthesis and exoskeleton. In this paper, a series elastic actuator (SEA) based ankle joint emulator and reflex-like control algorithm are purposed to test human push-recovery mechanism. The SEA element provides an easy way for ankle joint torque control. Proportion-Derivative (PD) based reflex-like control algorithm calculates reference torque for SEA element. Experimental results indicate that this proposed emulator has the ability to mimic human ankle joint quiet standing push-recovery performance. The emulator is a useful platform to test further human ankle joint torque control function.
\end{abstract}

Keywords: ankle joint, push-recovery, series elastic actuator, reflex-like control.

\section{INTRODUCTION}

Ankle joint has the function of maintaining balance while quiet standing. When the body is disturbed to lean forward, ankle muscles provide opposite torque to restore the human body to the balance position [1]. The network of neurons controls the extensors and flexors muscles in the process of quiet standing and push-recovery [2]. The output torque of the ankle joint is related to its rotation state. In 2001, the push-recover model for ankle joint established by WINTER pointed out that the ankle muscles have the simple stiffness characteristics of the spring [3]. According to the experiment for THOMAS in 1988, after human body is disturbed, the condition of muscle activation during reflex control nearly completely independent of the amplitude of stretch in the range from 2 to 7 degrees [4]. The relationship between the output torque and rotation angle is approximately linear.

The EMG signals of ankle muscles during push-recover process show that the output torque and rotation angle of ankle joints have an approximate linear relationship, but there are still significant deviations [5]. The joint proprioception, vestibular organs in the inner ear, and vision information are transferred to the network of neurons center for decision of the behavior of ankle joint [6]. On this basis, an optimal state estimation model for ankle position balance control was proposed in 2005 [7]. In 2006, the experiment by Kei Masani evidence a feedback proportional-derivative (PD) controller can effectively generate a desired preceding motor command controlling balance during quiet standing [8].

On the other hand, since Pratt put forward SEA in 1995 [9], SEA is widely applied for robot ankle joint. The SEA acts like mechanical "muscles" and allow the robots to interact softly with the world much like animals. In 2012, P.Cherelle at the Vrije Universiteit Brussel, Belgium, developed the Ankle Mimicking Prosthetic Foot (AMP-Foot 2.0), it successfully uses SEA to store and release energy during the push-recover process [10]. In 2014, Herr use a lower-power clutch in parallel with the SEA (CSEA) in a powered knee prothesis design [11]. The CSEA knee provided biomechanically accurate torque-angle behavior during walking. Beside of that, Sato et al also use the pneumatic actuators in power assist control for walking motion [12] [13].

In this paper, the second section introduces the mechanical structure of the robot ankle joint. In third section, first, make the human ankle joint muscle reflex experiment. Then the simulation of the muscle reflex mechanism of the ankle joint was realized by the muscle-like reflex control algorithm. The fourth section carried out the ankle rotation experiment with constant stiffness and the muscle like reflex control experiment. The fifth section summarizes the effect of the muscle-like reflex control algorithm combined with robot ankle structure in this paper.

\section{Mechanical Structure of ANKLE JoINT}

The human ankle plays an important role in both walking and standing. In the walking, the main function is providing sufficient energy for the body to move. While standing ankle has the function of resisting disturbance to maintain balance of human body. The robot ankle joint should be simulated on the shape and function of the human ankle joint. Our laboratory has completed the mechanical structure design of the SEA based Ankle Joint Emulator (SEA-AJE). Fig. 1 is the 3D model of SEA-AJE.

This work was supported by the National Natural Science Foundation of China under Grant [61603284] and the Excellent Dissertation Cultivation Funds of Wuhan University of Technology. 


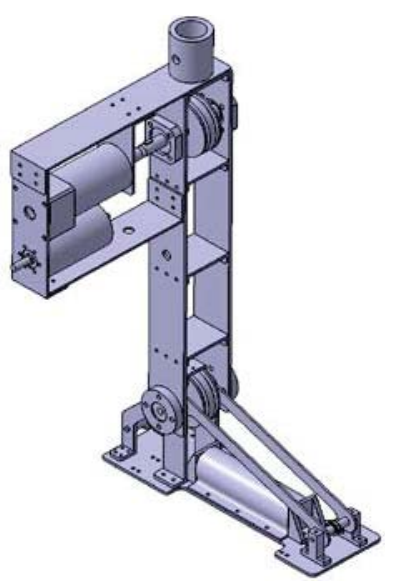

Fig. 1. 3D model of SEA-AJE

SEA-AJE includes a foot part and a shank part. The foot part has two springs with a slider between them, the shank part include two lower leg plates. The spring nearly toe is called pushoff spring (PS). It storages the energy from actuator and gravity during the dorsiflexion phase of stance. The spring nearly heel is called heel spring (HS). It storages energy from actuator and gravity during plantar flexion.

As the size is limited, motor and reducer are mounted on the proximal side of shank. They are connected by belt pulley. Steel wire rope is utilized to transmit power from reducer to ankle joint. As mentioned before, there are two springs PS and HS at the foot frame to form the series elastic element.

According to the results from Winter [14], a normal human foot is nearly $240 \mathrm{~mm}$ long and $80 \mathrm{~mm}$ wide. The human shank length is $450 \mathrm{~mm}$ and maximal diameter is $90 \mathrm{~mm}$. Therefore the SEA-AJE foot has been designed as $238 \mathrm{~mm}$ long and $78 \mathrm{~mm}$ wide. The detail message ankle structure are given in Table.2.

The rotary part of ankle joint is called Ankle Rotator (AR). Its center is connected with spindle (S1) through a key. Wire ropes bypass both sides of AR. It slides along the groove while the shank and motor rotate. Given that the human ankle joint diameter is about $50 \mathrm{~mm}$, the diameter of AR is $50 \mathrm{~mm}$ and thickness is $18 \mathrm{~mm}$. Two bearing adjusting rings $\mathrm{R} 1$ and $\mathrm{R} 2$ were put on both sides to lock AR, preventing moving through the S1. On the other side of R1 and R2, there are two small ball bearing $\mathrm{B} 1$ and $\mathrm{B} 2$. Its function is to connect $\mathrm{S} 1$ with support component while keeps S1 working as a rotating member. On the other side of $\mathrm{B} 1$ and $\mathrm{B} 2$, there is another bearing adjusting rings $\mathrm{R} 3$ and $\mathrm{R} 4$.

The direct stress value for $\mathrm{S} 1$ is calculated as follow:

$$
F_{\max }=\frac{1}{4} \pi D^{2} \sigma
$$

where, $D$ is the diameter of S1, $F_{\max }$ is the maximum force that can be borne for S1, $\sigma$ is yield strength for $\mathrm{S} 1$. S1 is made by SUS340, the biggest pressure for it could bear is $5765.04 \mathrm{~N}$. This value is far beyond the actual force.

The ankle joint and sole of the feet are shown in Fig.2. The PS and HS are placed in a spring box BX. At both sides of BX there are two baffles used to barrier spring movement. There is a through-hole in the baffle to let the wire rope pass through.

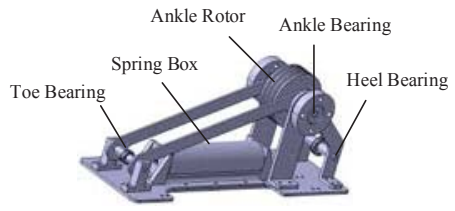

(a)

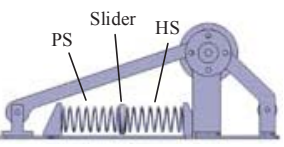

(b)

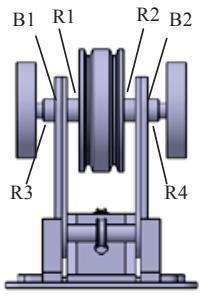

(c)

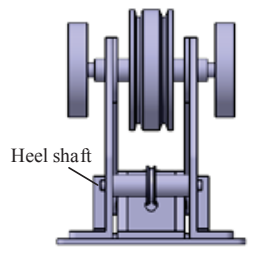

(d)
Fig. 2. Biped Robot Foot Structure

This ankle joint structure storages energy in springs while human leaning forward and release it during recover process. The force comes from motor acts on spring while the force from human body or outside acts on spring too. Springs deform when they are subjected to pressure and the deformation is proportional to the pressure. Because the spring can reduce the impact force, the instantaneous force of the motor will be reduced, and the reaction time demand for the motor will be reduced too.

Considering a normal human with $65 \mathrm{~kg}$ weight and $175 \mathrm{~mm}$ tall, the height of center of mass is calculated as follow:

$$
h_{g}=0.161 \cdot 0.553 \cdot 0.53 \mathrm{~h}+0.678[0.626 \cdot 0.52+(1-0.52)] \mathrm{h}
$$

where $h_{g}$ is height of gravity center, $\mathrm{h}$ is the height of human body.

Calculated by equation (2), the height of COM is $1.1209 \mathrm{~m}$. The torque produced by the human gravity at the ankle joint is calculated as follow:

$$
T_{a}=M g\left(h_{g}-\mathrm{h}_{l}\right) \sin \theta
$$

where, $M$ is the weight for experimenter, $\theta$ is ankle rotation angle, $T_{a}$ is the ankle joint torque of human gravity, $\mathrm{h}_{1}$ is the height of $\mathrm{S} 1$, its value is $70 \mathrm{~mm}$. Calculated by equation (3), $T_{a}$ is $35.03 \mathrm{Nm}$. The torque for everyone ankle joint is $17.52 \mathrm{Nm}$.

The moment arm of spring tension in SEA-AJE is equal to ankle radius. Thus the spring tension is calculated as follow:

$$
F_{s}=\frac{T_{a}}{R}
$$

where $\mathrm{R}$ represents robot ankle radius. $F_{s}$ represents spring tension. Calculated by the equation (4), $F_{S}$ is $700.8 \mathrm{~N}$. 
In this paper, DAI springs (Medium load spring, DAIspring, Japan) are used as SEA springs PS and HS. The detailed information for this spring is listed in Table.1.

TABLE I. SPRING PARAMETER.

\begin{tabular}{|c|c|c|c|c|}
\hline $\begin{array}{c}\text { Inner } \\
\text { Diameter } \\
(\mathbf{m m})\end{array}$ & $\begin{array}{c}\text { Outer } \\
\text { Diameter } \\
(\mathbf{m m})\end{array}$ & $\begin{array}{c}\text { Length } \\
(\mathbf{m m})\end{array}$ & $\begin{array}{c}\text { Stiffness } \\
(\mathbf{N} / \mathbf{m m})\end{array}$ & $\begin{array}{c}\text { Maximum } \\
\text { Compression } \\
(\mathbf{m m})\end{array}$ \\
\hline 12.5 & 25 & 50 & 76.59 & 16.0 \\
\hline
\end{tabular}

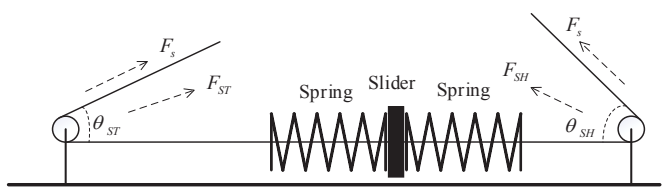

Fig. 3. Force analysis diagram of rotating shaft

At tip of the toe and heel there are two shafts respectively used to bypass steel wire rope. The shaft in tip of the toe is called ST. A bearing is installed in the middle of ST to let the steel wire rope pass through. In order to keep the bearing on the shaft, the bearing bushing is installed on both side of the bearing. The shaft in heel is called SH, its surrounding structure is similar to $\mathrm{ST}$. The force analysis diagram is shown in Fig.3. The pressure of ST and SH is calculated as follows:

$$
F_{a}=2 F_{s} \cos (\alpha / 2)
$$

where $\alpha$ is the angle between the two sides of the wire rope of the shaft. $F_{a}$ is the resultant force. Angle of the wire rope on toe and heel is 24 with 53 degrees, respectively.

Calculated by equation (5), the resultant force in toe is $1370.97 \mathrm{~N}$, the resultant force in heel is $1254.34 \mathrm{~N}$. In order to avoid the tension of the wire rope destroying the shafts, the diameters of toe and heel shafts are $4 \mathrm{~mm}$ and $6 \mathrm{~mm}$. The detailed information for these structural parts is shown in Table.2.

A normal human lower leg length is $480 \mathrm{~mm}$. So the robot shank structure has been designed as $480 \mathrm{~mm}$ long. The main body of the robot calf is two side plates. It was made by aluminum alloy. The leg plate end was connected with spindle $\mathrm{S} 1$ at the robot ankle joint position, and the top is connected with the iWalk2.0 in the position of the knee joint.
In order to strength the stability of the robot leg, three connectors are added between the leg plates. The SEA-AJE leg structure is shown in Fig.4.

In order to transfer the torque from reducer to the spring, the Knee Rotator (KR) is connected by the coupling and shaft S2 at the output end of the reducer. At the lower leg both sides are provided with two bearing with seats for fixed S2. The shaft diameter is $10 \mathrm{~mm}$ and its length is $90 \mathrm{~mm}$. KR is identical to AR, but the central bore diameter is $10 \mathrm{~mm}$. A fastening part is mounted on KR for fixing the wire ropes. The wire ropes are connected to the slider bypassing the slot on AR and KR.

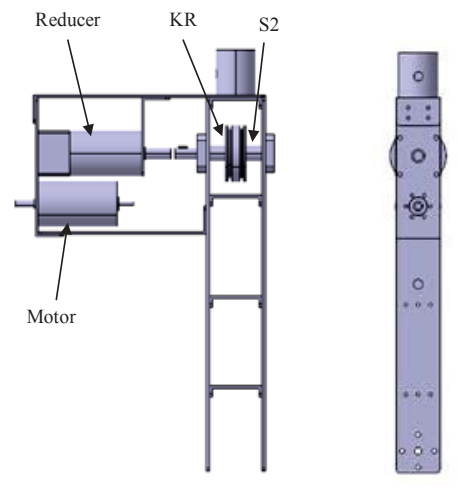

Fig. 4. Robot leg structure

The reducer output torque is calculated as follows:

$$
T_{o}=T_{m} \mathrm{P} \frac{\mathrm{n}_{2}}{\mathrm{n}_{1}}
$$

where, $T_{o}$ is output torque from reducer, $T_{m}$ is output torque from motor, $\mathrm{n}_{1}$ is the belt gear number in motor side, $\mathrm{n}_{2}$ is the belt gear number in reducer side, $\mathrm{P}$ is reduction ratio of reducer.

In this paper, the maxon motor RE40 (RE40, maxon motor, Swiss) is selected to provide power for robot ankle joint. Its stall torque is $2420 \mathrm{mNm}$. The reducer is PLE40 (PLE40, NEUGART, Germany), with reduction ratio 60 . The belt pulleys in motor and reducer side gear number is $\mathrm{n}_{1}=36, \mathrm{n}_{2}=40$, so the total reduction ratio $P_{r}$ is 66.67. Calculated by the equation (6), the maximum output torque is $161.34 \mathrm{Nm}$.

TABLE II. PARAMETER OF DETAILS.

\begin{tabular}{|c|c|c|c|c|c|c|}
\hline Part & $\begin{array}{c}\text { Length } \\
(\mathrm{mm})\end{array}$ & $\begin{array}{c}\text { Width } \\
(\mathrm{mm})\end{array}$ & $\begin{array}{c}\text { Thickness } \\
(\mathrm{mm})\end{array}$ & $\begin{array}{c}\text { Inner } \\
\text { Diameter } \\
(\mathrm{mm})\end{array}$ & $\begin{array}{c}\text { Outer } \\
\text { Diameter } \\
(\mathrm{mm})\end{array}$ & $\begin{array}{c}\text { Diameter } \\
(\mathrm{mm})\end{array}$ \\
\hline Foot & 238 & 78 & 2.5 & $/$ & $/$ & $/$ \\
\hline Leg Plate & 333 & 39 & 3 & $/$ & $/$ & $/$ \\
\hline AR & $/$ & 18 & $/$ & 6 & 50 & 6 \\
\hline S1 & 70 & $/$ & $/$ & $/$ & $/$ & $/$ \\
\hline R1\&R2 & 5 & $/$ & $/$ & 6 & 9 & $/$ \\
\hline R3\&R4 & 4.5 & $/$ & $/$ & 6 & 13 & 4 \\
\hline B1\&B2 & $/$ & 5 & $/$ & 6 & $/$ & 6 \\
\hline ST & 50 & $/$ & $/$ & $/$ & $/$ & \\
\hline SH & 42 & $/$ & & & & \\
\hline
\end{tabular}




$$
P_{r}=P \frac{n_{2}}{n_{1}}
$$

Encoder is used to collect the motor and ankle rotation angle information. The encoder E1 in Maxon motor is 1000 lines while the encoder E2 in robot ankle joint is 2000 lines. According to the values of encoder E1 and E2, the amount of spring compression can be calculated as follows:

$$
L_{s}=\left[\theta_{a}-\frac{\theta_{m}^{r}}{P} \times \frac{n_{1}}{n_{2}}\right] \mathrm{R}
$$

where, $L_{s}$ is spring compression length, $\theta_{a}$ is ankle rotation angle, $\theta_{m}^{r}$ is motor rotation angle.

\section{CONTROL PRINCIPLE AND AlgORITHM}

According to the muscle reflex control [15], the degree of muscle activation is related to angle and angular velocity. The relationship between them is similar with the PD model. Normally speaking, there is a corresponding relationship between muscle activation and muscle tension, which can be described by a Hill-based muscular model with strongly nonlinear. According to our experimental data for human pushrecover, the relationship between muscle activation and muscle tension for human ankle joint is certain. There is a PD control relationship between the muscle tension and the angle and angular velocity of the joints. The Fig.5 is shown the EMG signals for PD control and the original.

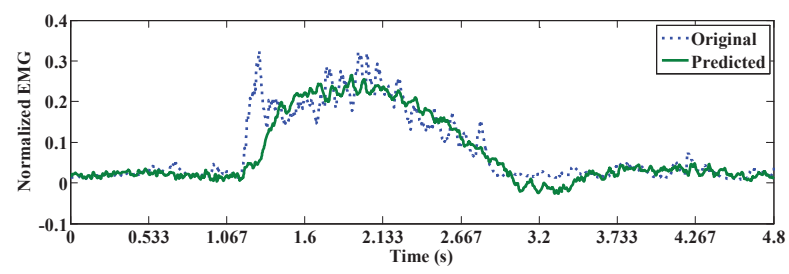

Fig. 5. Prediction results with linear equation

The human ankle joint output torque during push-recover process is shown in Fig.6, it can be simulated by a PD control model. Albert $\mathrm{H}$. Vette also adopts the approximate simulation method in studying the output torque of the ankle during quiet stance [16].

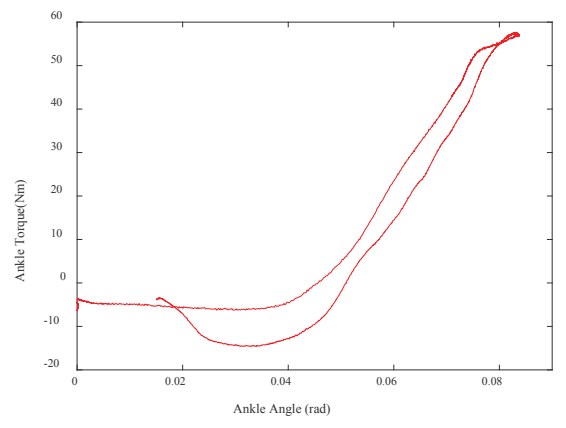

Fig. 6. Human ankle joint output torque curve

Thus, an ankle joint reflex control algorithm is designed for SEA-AJE. The reflex control is used as the outer loop of the control system to calculate the desired torque. Meanwhile, SEA control algorithm is used as the internal loop of the control system to maintain the stability of the ankle joint at any angle. The algorithm control block diagram is shown in Fig.7.

In this paper, the linear coefficient for ankle muscle is assumed as Virtual Stiffness (VS). The VS determines the desired output torque of the ankle at a certain rotation angle. The relationship for output torque and VS as follows:

$$
\begin{aligned}
& F_{s}=K_{v s} \theta_{a} R+C \dot{\theta}_{a} \\
& \tau_{s}=F_{s} R
\end{aligned}
$$

where, $K_{v s}$ is the VS for ankle joint, $F_{s}$ is the spring tension in ankle joint, $\theta_{a}$ is rotation angle of ankle joint, $C$ is the coefficient for ankle angle change rate, $\dot{\theta}_{a}$ is ankle angle change rate, $\tau_{\mathrm{s}}$ is the output torque for robot ankle.

In quiet standing state, the angular velocity of robot ankle is zero. The influence of ankle joint angular velocity can be ignored when calculating VS under quiet standing state. Calculated by equation (10), the idea VS value is $535371 \mathrm{~N} / \mathrm{m}$.

The motor and reducer pull the spring through the wire rope, and the pulling force produces the rotation torque on the ankle joint. When the robot ankle rotates, the motor will rotate to compression the spring to output torque. The value for spring tension Fs is calculated as follow:

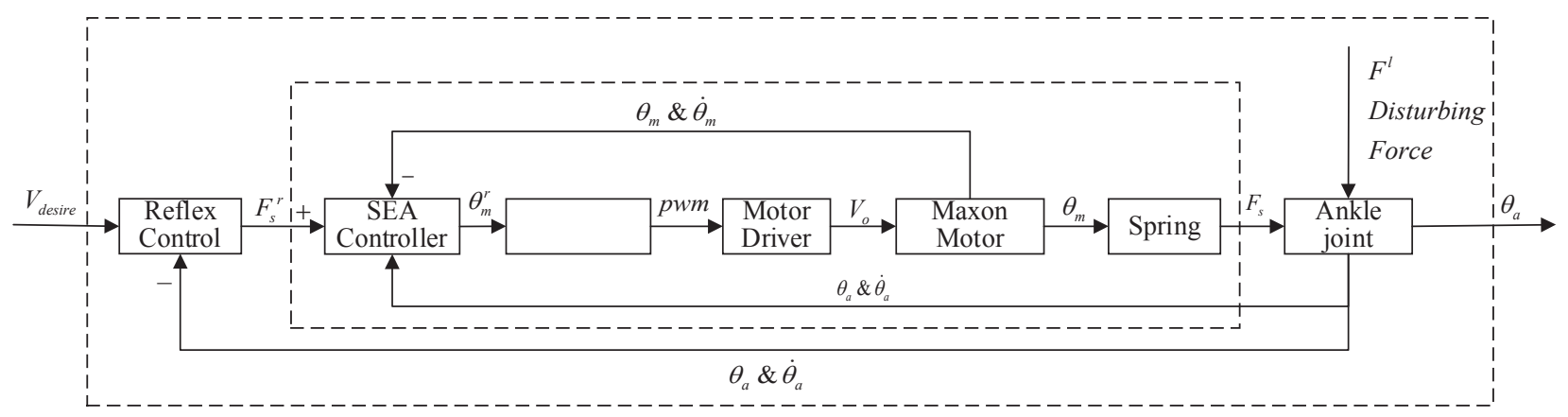

Fig. 7. Reflex Control Block Diagram 


$$
F_{s}=K_{s}\left(\theta_{a}-\frac{\theta_{m}^{r}}{P_{r}}\right)
$$

where $K_{s}$ represent the spring stiffness. According to the equations (9) and (11), the relationship between the rotation angle of motor, the rotation angle and VS as follows:

$$
\theta_{m}^{r}=\left(1-\frac{K_{v s}}{K_{s}}\right) \theta_{a} P_{r}+D \dot{\theta}_{a}
$$

where

$$
D=-\frac{C}{K_{s}} P_{r}
$$

It is the rotation angular velocity coefficient.

That motor angle $\theta_{m}^{r}$ will be used as the expected value in next inner loop. The inner loop is for position control. Motor is the main controlled object in this link. The purpose of inner loop is to rotate the motor as soon as possible to the desire angle and tries to avoid over-shoot. In order to achievement this goal, a feedback PD controller is implemented.

$$
V_{o}=\mathrm{p}\left(\theta_{m}-\theta_{m}^{r}\right)+\mathrm{d} \dot{\theta}_{m}
$$

where $\mathrm{p}$ is the proportionality coefficient, $\mathrm{d}$ is the differential coefficient, $V_{o}$ is the voltage output to the motor, $\theta_{\mathrm{m}}$ is current motor angle, $\dot{\theta}_{m}$ is angle change rate of motor.

\section{EXPERIMENTAL RESULTS AND DISCUSSION}

\section{A. Ankle rotation experiment with constant stiffness}

To test the effect of an imitated human ankle muscle reflex controller, the relationship between the output torque and the angle of robot ankle joint during rotation need be test.

As shown in Fig.8, the SEA-AJE be fixed on the tooling platform.

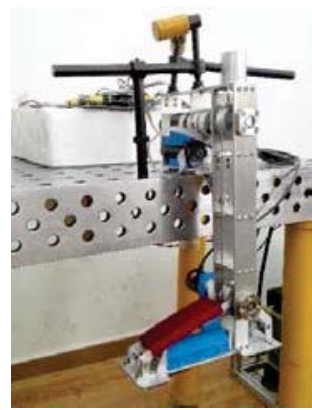

Fig. 8. SEA-AJE be fixed on the platform

After the SEA-AJE is fixed, only the foot can be rotated. The VS are set up for $191475 \mathrm{~N} / \mathrm{m}, 382950 \mathrm{~N} / \mathrm{m}$ and $571361 \mathrm{~N} / \mathrm{m}$. Corresponding torque coefficients are $118.6 \mathrm{Nm} / \mathrm{rad}, 240 \mathrm{Nm} / \mathrm{rad}$ and $357 \mathrm{Nm} / \mathrm{rad}$, respectively. In this experiment, the ankle joint rotated from the upright equilibrium position to the direction of plantar flexion, and the amount of spring compression at every angle of 0.1 degrees was recorded until the rotation angle to 5.6 degrees. Then the robot's foot was rotated to direction of dorsiflexion, as same with the plantar flexion.

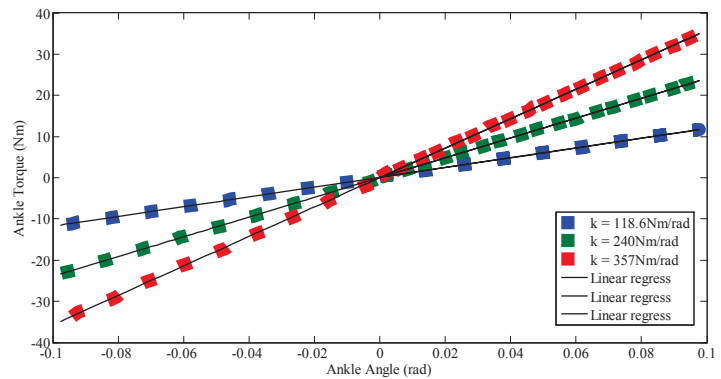

Fig. 9. Torque curve of robot ankle joint under fixed stiffness condition

The experimental results are shown in Fig.9. The torque value of the longitudinal coordinate for ankle joint is the product of spring compression and force arm of the ankle joint and the spring stiffness. The three curves drawn from the experimental data indicate that output torque of robot ankle joint is basically linear with the rotation angle under steady state, and the linear coefficient is basically consistent with the set value.

\section{B. Muscle-like reflex control experiment}

In this experiment, the subject is experiencing the process of forward and backward balance after being pushed from the back. The experimenter is $175 \mathrm{~cm}$ height and $65 \mathrm{KG}$ weight. The SEAAJE plays the role of human ankle joint.

The mechanical structure at top of SEA-AJE consists of a bolt. Through the bolt, iwalk 2.0 is connected to SEA-AJE. The experimental device is placed vertically, the foot floor of SEAAJE is in contact with the ground. The experimenter put his lower leg on the iwalk2.0 protruding plane and his thigh was closed with iwalk 2.0 vertical column. The experimenter's lower leg and thigh were fixed on iwalk 2.0 by bandage.
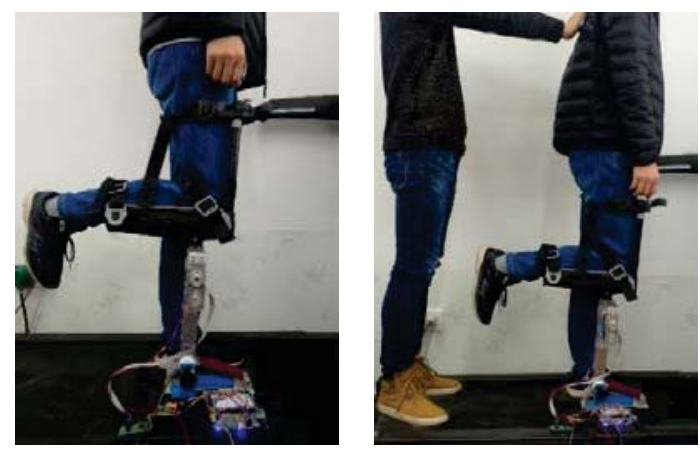

Fig. 10. (a)Experimenter quiet stand. (b)Experimenter be pushed from back.

From another experimenter, a short thrust was applied to the experimenter's back. After being pushed, the experimenter contraction of his left leg muscle to make body eventually return to the balance position. At the same time, SEA-AJE also works to keep the body balance. In addition, the thrust for experimenter should be in the acceptable range for human. 
In push-recover process, the angle information collected by encoders, and be processed by STM 32 controller get the spring compression length. Then the compression and ankle angle massage be transmitted to host computer through WIFI. In this experiment, the frequency of sampling and calculation is $20 \mathrm{KHz}$.

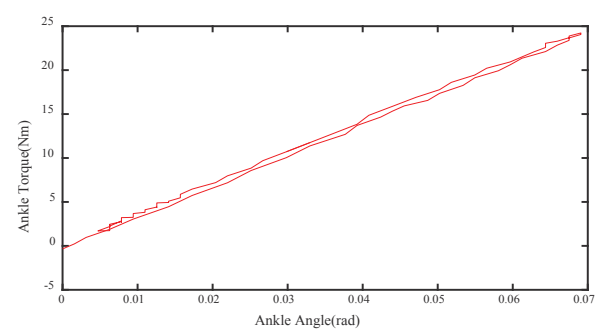

Fig. 11. Torque angle relation curve of muscle-like reflex control

The relationship between the output torque and robot ankle angle in the muscle imitated reflex control experiment is shown in Fig.11. The torque of the robot ankle joint is zero when the inclination angle of the human body is zero. When the human body leaned forward, the output torque of SEA-AJE is basically proportional to the ankle joint angle. There is a certain distance between the moment curves in the process of forward and recovery. This was caused by the differential lag in the imitated muscle reflex control link. When the body inclination angle reaches 3 degrees, the output torque from robot ankle joint is $18.25 \mathrm{Nm}$, and the spring tension is $730 \mathrm{~N}$. The relationship between the SEA-AJE output torque and the ankle joint angle is basically consistent with the human ankle joint muscle.

Compared the curves in Fig.11 and Fig.6, the output torque from SEA-AJE lacked the non-response stage. The reason is that human reflex control system need some time to response the disturbance, but the SEA-AJE controller haven't set delay time. Beside of that, the SEA-AJE output torque curve in forward and back is close than human. In this paper, we assumed that human ankle joint is a spring-damped system. Human need muscle supplied more power to get the ankle joint work, at the same time, it improved the human body stability.

\section{CONCLUSION}

In this paper, a new type of ankle joint emulator robot is designed. The structure uses SEA as an actuator and uses rope drive between the spring and reducer. According to the relationship between output torque and angle of human ankle joint during push-recover process, an muscle-like reflex control algorithm is designed to control the robot ankle structure.

Through the ankle rotation experiment with constant stiffness, the output torque characteristics for SEA-AJE in static state are tested. The muscle-like reflex control experiment shown the muscle-like reflex control curve slope is similar with the human in push-recovery process. The SEA-AJE worked like human ankle joint under the muscle-like reflex control. The difference between SEA-AJE and human ankle in the process of forward and recovery shows that there are still have some differences. It's because the muscle-like controller's differential lag not good like human muscle. And it caused the SEA-AJE have some mismatch with human leg, it's more instability.

\section{ACKNOWLEDGEMENTS}

This work was supported by the National Natural Science Foundation of China under Grant [61603284] and the Excellent Dissertation Cultivation Funds of Wuhan University of Technology.

\section{REFERENCES}

[1] M.J. Warnica, T.B. Weaver, S.D. Prentice, and A.C. Laing, "The influence of ankle muscle activation on postural sway during quiet stance." Gait \& posture, vol. 39, pp. 1115-1121, April 2014.

[2] H. Geyer, and H. Herr, "A muscle-reflex model that encodes principles of legged mechanics produces human walking dynamics and muscle activities." IEEE Transactions on neural systems and rehabilitation engineering, vol. 18, pp. 263-273, June 2010.

[3] D.A. Winter, A.E. Patla, S. Rietdyk, and M.G. Ishac, "Ankle muscle stiffness in the control of balance during quiet standing." Journal of neurophysiology, vol. 85, pp. 2630-2633, June 2001.

[4] T. Sinkjaer, E. Toft, S. Andreassen, and B.C. Hornemann, "Muscle stiffness in human ankle dorsiflexors: intrinsic and reflex components." Journal of neurophysiology, vol. 60, pp. 1110-1121, September 1988.

[5] T. Kawase, H. Kambara, and Y. Koike, "A power assist device based on joint equilibrium point estimation from EMG signals." Journal of Robotics and Mechatronics, vol. 24, pp. 205-218, February 2012.

[6] R. Fitzpatrick, D. Burke, and S.C. Gandevia, "Loop gain of reflexes controlling human standing measured with the use of postural and vestibular disturbances." Journal of neurophysiology, vol. 76, pp. 39944008, December 1996.

[7] A.D. Kuo, "An optimal state estimation model of sensory integration in human postural balance." Journal of Neural Engineering, vol. 2, S235, August 2005.

[8] K. Masani, A.H. Vette, and M.R. Popovic, "Controlling balance during quiet standing: proportional and derivative controller generates preceding motor command to body sway position observed in experiments." Gait \& posture, vol. 23, pp. 164-172, February 2006.

[9] G.A. Pratt, and M.M. Williamson, "Series elastic actuators." Intelligent Robots and Systems 95.'Human Robot Interaction and Cooperative Robots', Proceedings. 1995 IEEE/RSJ International Conference on, vol. 1, pp. 399-406, August 1995.

[10] P. Cherelle, A. Matthys, V. Grosu, B. Vanderborght, D. Lefeber, "The amp-foot 2.0: Mimicking intact ankle behavior with a powered transtibial prosthesis." Biomedical Robotics and Biomechatronics (BioRob), 2012 4th IEEE RAS \& EMBS International Conference on, pp.544-549, August 2012.

[11] E.J. Rouse., L.M. Luke, and H.M. Herr, "Clutchable series-elastic actuator: Implications for prosthetic knee design." The International Journal of Robotics Research, vol. 33, pp. 1611-1625, October 2014.

[12] M. Sato, E. Yagi, and K. Sano, "Power Assist Control Calculated by a Human Model and Joint Angles for Walking Motion Using Pneumatic Actuators." JRM, vol. 25, pp. 915-922, December 2013.

[13] T. Onodera, E. Suzuki, M. Ding, H. Takemura, and H. Mizoguchi, "Force, Stiffness and Viscous Damping Control of a Stewart-Platform-Type Ankle-Foot Rehabilitation Assist Device with Pneumatic Actuator." Journal of Robotics and Mechatronics, vol. 25, pp. 897-905, December 2013.

[14] D.A. Winter, Biomechanics and motor control of human movement. 4th ed. Hoboken: John Wiley \& Sons, 2009, pp.86-90.

[15] N. Thatte, H. Geyer, "Toward balance recovery with leg prostheses using neuromuscular model control." IEEE Transactions on Biomedical Engineering, vol. 63, pp. 904-913, August 2016.

[16] A.H. Vette, K. Masani, and M.R. Popovic, "Implementation of a physiologically identified PD feedback controller for regulating the active ankle torque during quiet stance." IEEE Transactions on Neural Systems and Rehabilitation Engineering, vol. 15, pp. 235-24, June 2007. 